\title{
Comparison between Minimum Power Consumption and Minimum Battery Cost Routing for Energy Management in Wireless Ad Hoc
}

\author{
Vijay Gohel \\ Department of electronics and \\ communication \\ L.D. College of engineering \\ Ahmedabad,India
}

\author{
Kishor G. Maradia, Ph.D \\ Department of electronics and \\ communication \\ Government Engineering \\ College \\ Modasa,India
}

\author{
Usha Neelakantan \\ Department of electronics and \\ communication \\ L.D. College of engineering \\ Ahmedabad,India
}

\begin{abstract}
Nodes in wireless Ad hoc network have fixed battery life time. If nodes are used continuously for transmission of data packets, extra energy is needed by that node and after proper amount of time the energy level may not be sufficient for data transmission resulting in link failure. In this paper, two energy routing algorithm-Minimum power consumption Routing (MPCR) \& Minimum Battery cost Routing (MBCR) have considered and studied their performances in terms of power required for the same network geometry. Simulations are carried out using MATLAB. Finally from the simulation results concluded that MBCR consumes less overall power as compare to MPCR.
\end{abstract}

\section{General Terms}

Energy efficiency, WANETS, Routing Algorithm.

\section{Keywords}

MPCR , MBCR , Energy management, Power consumption.

\section{INTRODUCTION}

Communication between the lots of devices makes it possible to innovate services. This inter devices communication are very powerful and complex too. In the past few years, many wireless connectivity standards and technologies have been comes out. These technologies make available to connect extended range of computing and telecommunications devices fairly and simply, without the need to purchase, carry, or connect cables. These technologies give opportunities for speedy ad hoc connections and the possibility of automatic connections between equipment. Ad hoc wireless network can be defined as the wireless networks that use multi hop radio relaying and capable of operating without the support of any fixed infrastructure. The void of any central managing support makes routing of data more hard in ad hoc as compare to other wireless ad hoc network i.e. Cellular network. The routing and the resource management in ad hoc wireless network are done in the distributed way such that each node coordinates to communicate by itself. These makes each node to be more advanced. It needs a nodes function both as a router for routing packets and as network host for transmitting data.

In this paper, considered the problem of routing in a wireless ad hoc network from power efficiency point of view. In this simulated minimum power consumption routing (MPCR)[1] $\&$ minimum battery cost routing (MBCR)[1] and studied their performances in terms of overall power consumption for the studied their performances in terms of overall power consumption for same network geometry. The rest of the paper is organized as follows: Section 2 presents the related work. Section 3 discusses both the protocols MPCR \& MBCR in brief. Section 4 presents the simulation setup, Section 5 gives the results and Section 6 concludes the paper.

\section{RELATED WORK}

In [1] authors explains that To maximize the lifetime of ad hoc mobile networks, the power consumption rate of each node must be evenly distributed, and the overall transmission power for each connection request must be minimized. These two mottoes cannot be satisfied simultaneously by applying routing algorithms proposed in previous work. In this article they give a new power-aware routing protocol to satisfy these two restrictions simultaneously; they also compare the performance of different types of energy-related routing algorithms via simulation. A simulation result verifies the need to target equality in attaining service availability performance of the whole network vs. the lifetime of ad hoc mobile devices. In [2] authors propose a cooperation-based routing algorithm, namely, the Minimum Power Cooperative Routing (MPCR) algorithm, which makes full use of the cooperative communications at a same time constructing the minimum-power route. The MPCR algorithm constructs the minimum-power route, which guarantees certain throughput, as a cascade of the minimum-power single-relay building blocks from the source to the destination. Thus, any distributed shortest path algorithm can be used to find the optimal cooperative route with polynomial complexity. Using analysis, they show that the MPCR algorithm can achieve power saving of $65.61 \%$ in regular linear networks and $29.8 \%$ in regular grid networks compared to the existing cooperationbased routing algorithms, where the cooperative routes are constructed based on the shortest-path routes. From simulation results, MPCR algorithm can have $37.64 \%$ power saving in random networks compared to those cooperation based routing algorithms. In [3] authors elaborates transmission energy management is proposed to minimize energy consumption of nodes in network. Transmission energy consumption is inversely proportional to remaining energy of node in network. NS2 simulation model is used to analysis energy requirement of nodes in network and results show that varying the transition and receiving energy makes impact on the remaining energy of nodes in network. An optimum remaining energy is required to maintain working of network. 


\section{DESCRIPTION OF ROUTING ALGORITHMS FOR WIRELESS AD HOC NETWORKS}

\subsection{Minimum Power Consumption Routing (MPCR)}

The power require to send a packet from one node to another is inversely proportional to the nth power of the distance between them, where $\mathrm{n}$ can take values from 2 or 4 depending upon the distance and terrain between the nodes. To transmit data from node ni to node nj successfully the SNR of node $j$ to be greater than the specific value $\Psi \mathrm{j}$

$$
S N R_{j}=\frac{G_{i, j} P_{i}}{\sum_{k \neq i} P_{k} G_{k, j}+\eta_{j}} \Psi_{j}(\mathrm{BER})
$$

Where $\mathrm{Pi}$ is the transmission power of host, $\mathrm{Gi}, \mathrm{j}=1 / d_{i, j}^{n}$ is the path gain between hosts ni and $n j, \eta_{j}$ is the thermal noise at host $\mathrm{nj}$ and $\psi \mathrm{j}$ is threshold value. Therefore, the minimum transmission power is dependent on interference noise, distance between hosts, and desired BER. To obtain the route with the minimum total power, the transmission power $P(n i$, $n j$ ) between hosts $n j$ and $n j$ can be used as a metric. Transceiver power (the power used when receiving data) as well as transmission power were considered as a cost metric, and the distributed Bellman-Ford algorithm was used [6]. At node $n j$, it computes the power cost

$$
C_{i, j}=P_{\text {transmit }}\left(n_{i}, n_{j}\right)+P_{\text {transceiver }}\left(n_{j}\right)+\operatorname{Cost}\left(n_{j}\right)
$$

where $n i$ is a neighbouring node of $n j, P$ transceiver $\left(n_{j}\right)$ is the transceiver power at node $n j$, and $\operatorname{Cost}\left(n_{j}\right)$ is the total power cost from the source node to node $n j$. This value is sent to node $n i$. Subsequently, at node $n i$ it computes its power cost by using the following equation:

$$
\operatorname{Cost}\left(n_{j}\right)=\min _{j \in N H_{(i)}} C_{i, j} \text {. }
$$

Where,

$$
N H_{(i)}=\left\{j, n_{j} \text { is the neighbour node of } n_{i}\right\}
$$

The path with minimum cost from the source node to node $n i$ is selected. This procedure is repeated until the destination node is reached.

\subsection{Minimum battery cost routing (MBCR)}

In this individual battery charges are taken in to consideration, The path selected must not contain the nodes that have least battery capacity.

Let $c_{i}^{t}$ be the battery capacity of a host $n i$ at time $t$ ranging between 0 and 300 . We define $f\left(c_{i}^{t}\right)$ as a battery cost function of a host $n i$.

$$
f_{i}\left(c_{i}^{t}\right)=\frac{1}{c_{i}^{t}}
$$

Now, suppose a node's willingness to forward packets is a function of its remaining battery capacity. The less capacity it has, the more reluctant it is. As proposed, one possible choice for $f i$ is As the battery capacity decreases, the value of cost function for node $n i$ will increase. The battery cost $R j$ for route $i$, consisting of $N$ nodes is the sum of the cost functions of all these $\mathrm{N}$ nodes. Therefore, to find a route with the maximum remaining battery capacity, we should select a route $i$ that has the minimum battery cost.

$$
R_{i}=\min \left(R_{j}\right) \quad \forall \mathrm{j} \in \mathrm{A}
$$

Where, $\mathrm{A}$ is the set of routes from Source to Destination.

\section{SIMULATION BASICS}

In this simulation MATLAB R2013a (8.1.0.604) is used .It is high level language and interactive environment used by millions of engineers and scientists worldwide. It lets you explore and visualize ideas and collaborate across disciplines including signals and image processing, communications, control systems and computational finance. The parameters used for carrying out simulation are summarized in table 1 .

Table.1 Simulation parameters

\begin{tabular}{|c|c|}
\hline Parameter & Value \\
\hline Routing algorithm & MPCR,MBCR \\
\hline Terrain size & $200 * 200 \mathrm{~m}$ \\
\hline MAC layer & 802.11 \\
\hline No of nodes & $10-50$ \\
\hline Initial power & $10 \mathrm{~W}$ \\
\hline Transceiver power & $50 \mathrm{~mW}$ \\
\hline Coverage of node & $30 \mathrm{~m}$ \\
\hline
\end{tabular}

The goal of these simulation is to show which routing algorithm gives low power on the entire path calculated by considering particular algorithm.

\section{SIMULATION RESULTS}

In this simulation the first step is to generation of nodes. Following figure 1 and 2 shows the generation of nodes in matlab.

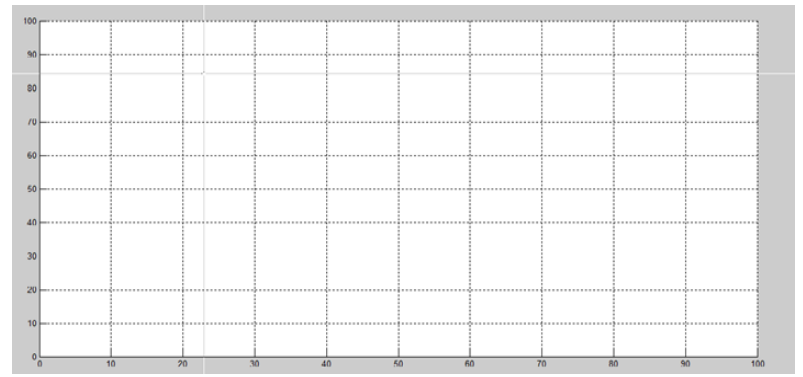

Figure 1

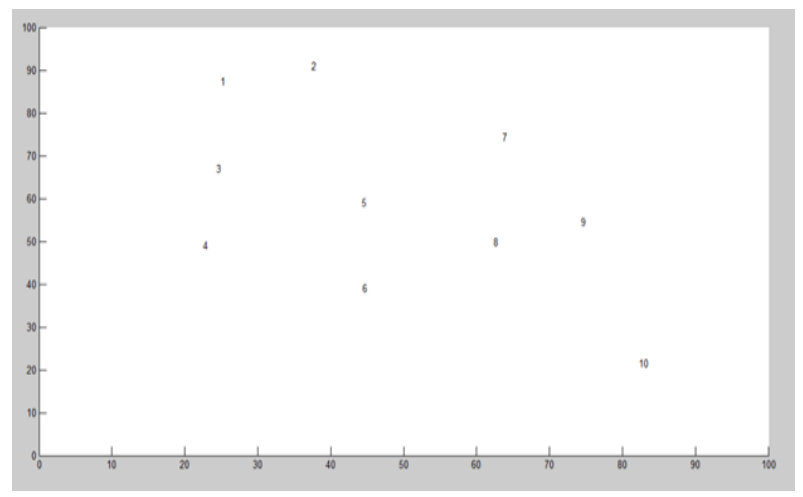

Figure 2

After, creating the node the main things is to generate shortest path algorithm to send data from source to destination. This is shown in figure 3 


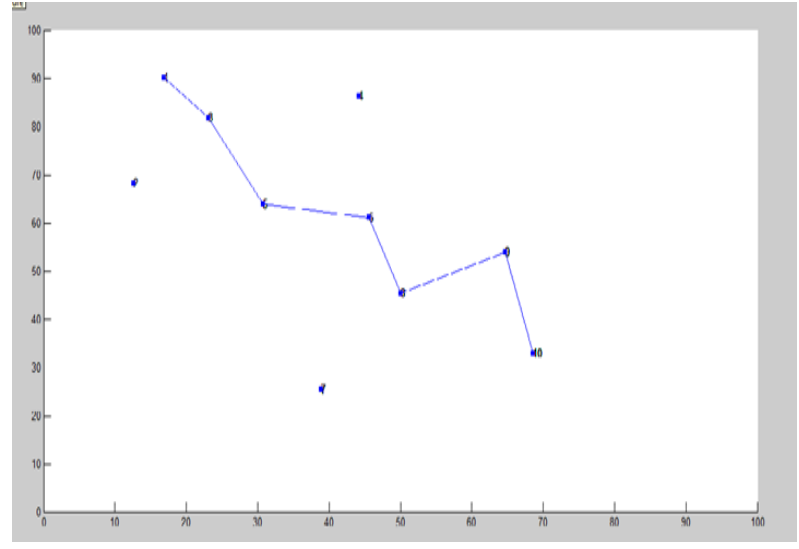

Figure 3

Then created minimum power consumption routing in matlab as shown in figure 4 .

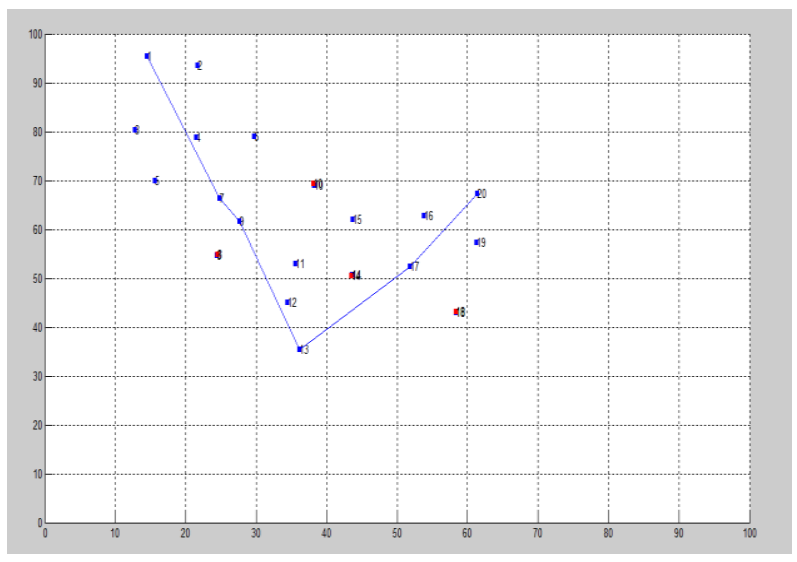

Figure 4

Then after created minimum battery cost routing in matlab as shown in figure 5

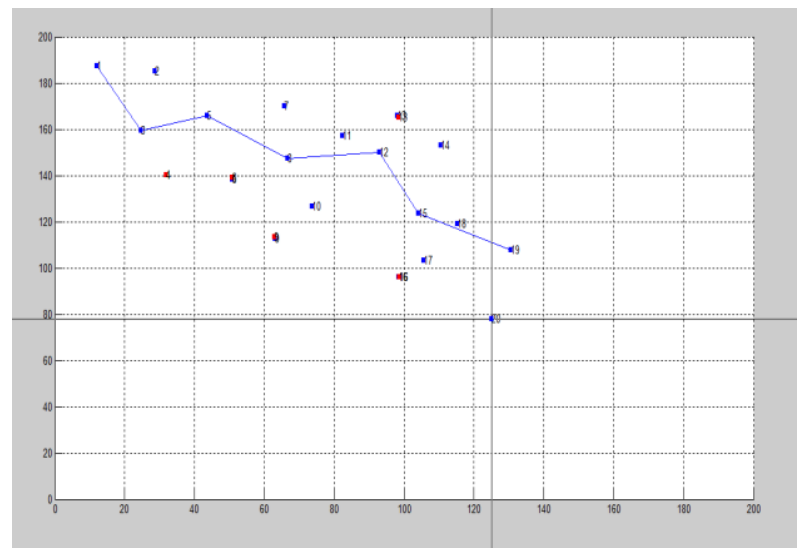

Figure 5

Then created various nodes topology from 10 to 50,5 nodes gap in between and applied both routing algorithm on same topology and calculated the power require for each one. All calculated power then plotted on graph as shown in figure 6,7 and 8 .

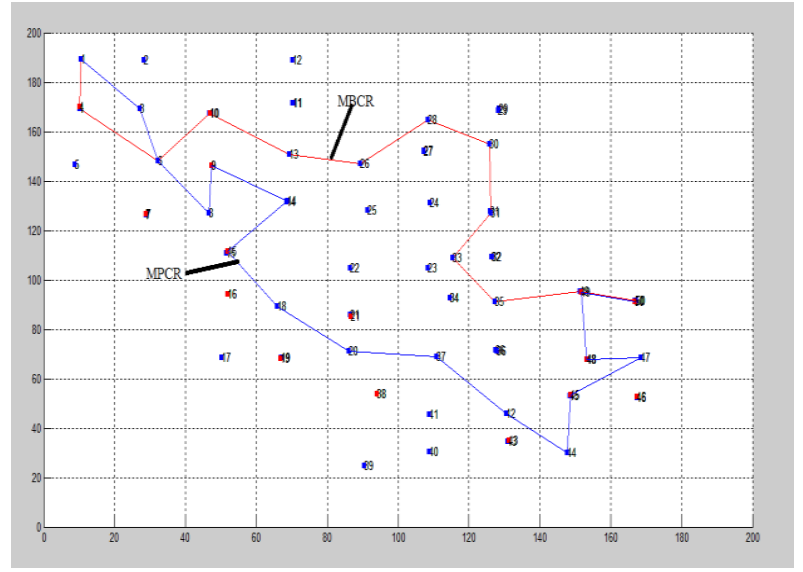

Figure 6

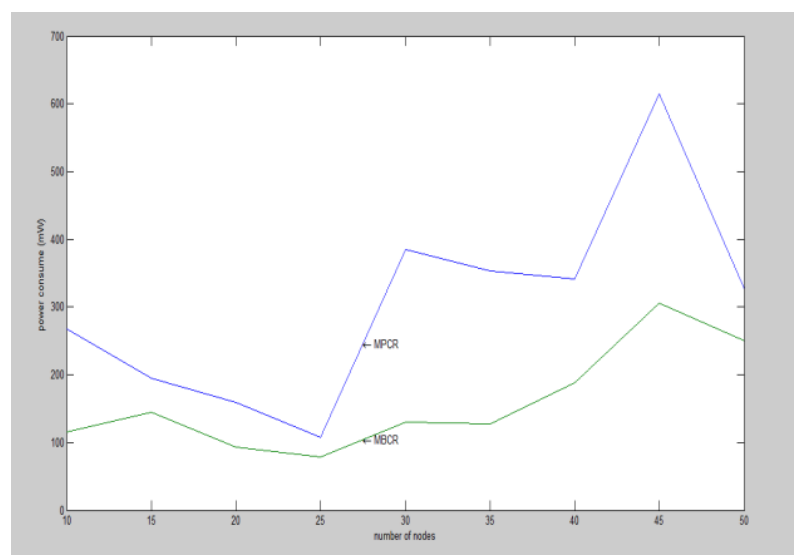

Figure 7

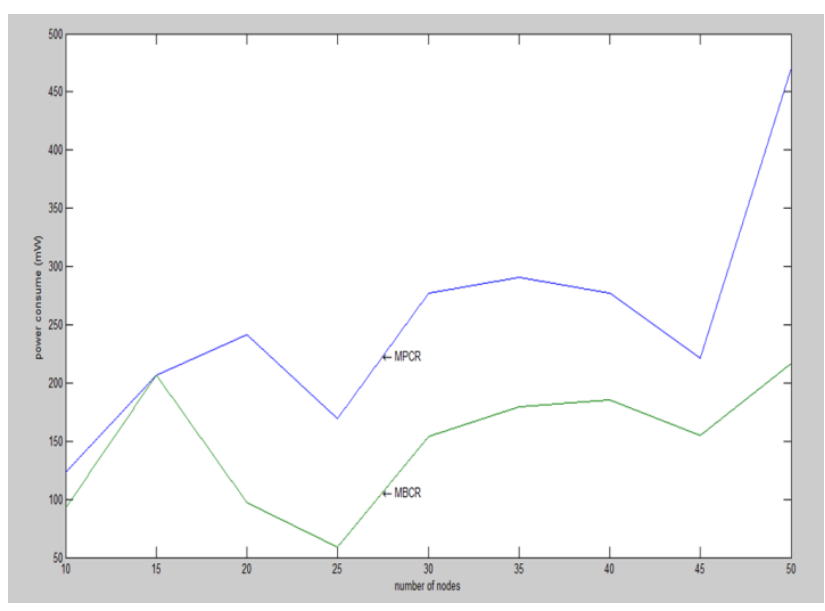

Figure 8

\section{CONCLUSIONS}

In this paper, mainly considered two routing algorithms MPCR \& MBCR and compared their performances in terms of power consumed from source to destination using MATLAB. From the simulations we observed that MBCR selects the route with nodes containing maximum battery value i.e., the route with maximum lifetime is selected. MPCR does not consider the lifetime of the network and chooses the route which requires less power cost. Thus, from the observations concluded that MBCR require less power compare to MPCR. 


\section{REFERENCES}

[1] C.-K. Toh, "Maximum battery life routing to support ubiquitous mobile computing in wireless ad hoc networks", IEEE Communications Magazine, June 2001, pp. 138-147.

[2] Ahmed S. Ibrahim, Zhu Han, and K. J. Ray liu. 'Distributed energy-efficient cooperative routing in wireless networks', IEEE transaction on wireless communication vol. 7, no. 10, october 2008 .

[3] Krishan Kumar Raman, Manav Rachna 'Transmission energy management for wireless ad-hoc network', 978-14 799-2995-5/14/\$31.00@2014IEEE.

[4] Humaira Nishat, Dr.D.Srinivasa Rao, Dr.Ch.Balaswamy, 'Energy Efficient Routing Protocols for Mobile Ad Hoc Networks',Internationa Journal of Computer Applications (0975-8887)Volume26-No.2,July2011.

[5] C.Siva Ram Murthy and B.S.Manoj, "Ad Hoc Wireless Networks Architectures and Protocols," Pearson Education Inc. p.no.600-623.
[6] S. Singh, M. Woo, and C. S. Raghavendra, "PowerAware Routing in Mobile Ad Hoc Networks," Proc. MobiCom'98, Dallas, TX, Oct. 1999

[7] E. Royer and C.-K. Toh, "A Review of Current Routing Protocols for Ad Hoc Mobile Wireless Networks," IEEE Pen Commun.vol.6,no.2,Apr.1999,pp.46-55.

[8] S:J. Lee, M. Gerla, and C.-K. Toh, "A Simulation Study of Table-Driven and On-Demand Routing Protocols for Mobile Ad Hoc Networks," IEEE Network, July 1999.

[9] C.-K. Toh, "Associativity Based Routing for Ad Hoc Mobile Networks," Wireless Pers. Commun. 1.. Special Issue on Mobile Networking and Computing Systems, vol.4,no.2,Mar.1997.

[10] C. Raghavendra. PAMAS S. Singh. Power aware multiaccess protocol with signalling for ad hoc networks. In SIGCOMM Computer Communication Review, volume 7, pages5-26,Dec1998. 